\title{
miRNAs can predict prostate cancer biochemical relapse and are involved in tumor progression
}

\author{
ANNIKA FENDLER ${ }^{1-3}$, MONIKA JUNG $^{2}$, CARSTEN STEPHAN $^{2}$, RICHARDSON J. HONEY $^{4}$, \\ ROBERT J. STEWART ${ }^{4}$, KENNETH T. PACE $^{4}$, ANDREAS ERBERSDOBLER ${ }^{5}$, \\ SARA SAMAAN ${ }^{6}$, KLAUS JUNG $^{2,3^{*}}$ and GEORGE M. YOUSEF ${ }^{6,7 *}$
}

\author{
${ }^{1}$ Department of Biology, Chemistry and Pharmacy, Free University; ${ }^{2}$ Department of Urology, University Hospital Charité; \\ ${ }^{3}$ Berlin Institute of Urologic Research, Berlin, Germany; ${ }^{4}$ Division of Urology, Department of Surgery, St. Michael's Hospital, \\ Toronto, Ontario, Canada; ${ }^{5}$ Department of Pathology, University Hospital Rostock, Rostock, Germany; ${ }^{6}$ Department of \\ Laboratory Medicine and the Keenan Research Centre in the Li Ka Shing Knowledge Institute St. Michael's Hospital; \\ ${ }^{7}$ Department of Laboratory Medicine and Pathobiology, University of Toronto, Toronto, Ontario, Canada
}

Received May 9, 2011; Accepted June 15, 2011

DOI: 10.3892/ijo.2011.1128

\begin{abstract}
Prostate cancer is the leading cancer diagnosed and the second most common cause of cancer related death in the western world. For prognostic monitoring after prostatectomy, recurrent increase of prostate-specific antigen (PSA), the so-called PSA or biochemical relapse remains the leading biomarker. There is currently no biomarker that can accurately predict the risk of relapse at the time of surgery. We analyzed formalin-fixed and paraffin-embedded tissue samples from 52 primary prostate cancers and normal adjacent tissues obtained after radical prostatectomy. Patients were grouped into two categories: i) patients with early biochemical relapse ( $<1$ year after radical prostatectomy) and ii) patients with late or no biochemical relapse ( $>2$ years after surgery). Multiplex real-time quantitative polymerase chain reaction (RT-qPCR) analysis was performed to identify a miRNA signature that can predict relapse. Results were validated by quantitative RT-PCR analysis. We identified 63 miRNAs that were differentially expressed among the same categories of patients, of whom 35 miRNAs were up-regulated and 28 were down-regulated. Literature search shows that many of these miRNAs have an established prognostic significance in other cancers and can be actively involved in tumor progression. Target prediction analysis showed that predicted targets of these miRNAs could
\end{abstract}

Correspondence to: Dr Klaus Jung, Department of Urology, University Hospital Charité, Schumannstr. 20/21, D-10117 Berlin, Germany

E-mail: klaus.jung@charite.de

\section{*Contributed equally}

Key words: microRNA, prostate cancer, prognostic marker, tumor marker, prostate cancer relapse, biochemical relapse, androgen, prostate-specific antigen be involved in biological processes and pathways that enhance tumor progression. We experimentally validated the role of one of the dysregulated miRNAs (miR-10b) in relapse using proliferation and wound-healing assay. miRNAs can be reliable predictive markers for biochemical relapse of prostate cancer at the time of radical prostatectomy. This should have significant impact on patient managing plans.

\section{Introduction}

Prostate cancer (PCa) is the most frequently diagnosed and the second most cause of cancer-related death in the Western world. The most reliable biomarker for $\mathrm{PCa}$ is prostate-specific antigen (PSA), which is helpful for screening, diagnosis and follow-up after surgery.

Approximately $15-30 \%$ of patients experience a biochemical relapse after curative treatment of radical prostatectomy (RP) or radiation therapy $(1,2)$. It is still a challenging task in $\mathrm{PCa}$ to identify patients with aggressive tumors to provide them with closer follow-up and more intensive treatment. For prognostic monitoring after prostatectomy or adjuvant therapy, a recurrent PSA increase, the so-called PSA or biochemical relapse, remains the only available marker. Yet, it has been shown that biochemical relapse is not inevitably leading to disease progression (3). Moreover, there is currently no biomarker that can accurately determine relapse risk preoperatively.

Various clinical models are established to estimate the risk of biochemical recurrence or clinical failure as has been reviewed recently (4), for example risk stratification models or risk scores. The accuracy of these models is limited as patients with heterogeneous disease characteristics will be clustered in the same risk group, thereby decreasing the predictive accuracy (4). As nomograms allow the use of weighted variables, they have a higher accuracy than classical models. However, most clinical prediction models still lack accuracy. Therefore, much effort is invested into identification of new more accurate prognostic markers for $\mathrm{PCa}$. 
Table I. Patients and tumor characteristics.

\begin{tabular}{|c|c|c|c|c|c|c|}
\hline \multirow[b]{3}{*}{ Age (years) } & \multicolumn{2}{|c|}{$\begin{array}{l}\text { Relapse }<1 \text { year } \\
\quad(\mathrm{N}=24)\end{array}$} & \multicolumn{2}{|c|}{$\begin{array}{l}\text { Relapse }>1-4 \text { years } \\
\qquad(\mathrm{N}=16)\end{array}$} & \multicolumn{2}{|c|}{$\begin{array}{c}\text { No relapse within } 3 \text { years } \\
(\mathrm{N}=12)\end{array}$} \\
\hline & Median (range) & \multirow[t]{2}{*}{$\mathrm{N}(\%)$} & Median (range) & \multirow[t]{2}{*}{$\mathrm{N}(\%)$} & Median (range) & \multirow[t]{2}{*}{$\mathrm{N}(\%)$} \\
\hline & $61 \quad(51-71)$ & & $63.5 \quad(49-73)$ & & $65.5 \quad(55-72)$ & \\
\hline Pre-operative PSA (ng/ml) & $8.9(0.8-25.0)$ & & $7.0(2.1-41.9)$ & & $6.5(3.7-25.8)$ & \\
\hline \multicolumn{7}{|l|}{ Pathological tumor stage } \\
\hline pT2a & & & & $1(6)$ & & \\
\hline pT2b & & $3(13)$ & & $4(25)$ & & $1(8)$ \\
\hline pT2c & & $5(21)$ & & $2(13)$ & & $4(33)$ \\
\hline pT3a & & $12(50)$ & & $6(38)$ & & $5(42)$ \\
\hline pT3b & & $4(17)$ & & $3(19)$ & & $2(17)$ \\
\hline \multicolumn{7}{|l|}{ Gleason score } \\
\hline 6 & & $3(13)$ & & $1(6)$ & & $3(25)$ \\
\hline 7 & & $13(54)$ & & $8(50)$ & & $5(42)$ \\
\hline 8 & & & & $2(13)$ & & $3(25)$ \\
\hline 9 & & $7(29)$ & & $5(31)$ & & $1(8)$ \\
\hline 10 & & $1 \quad(4)$ & & & & \\
\hline \multicolumn{7}{|l|}{ Surgical margins } \\
\hline R0 & & $15(63)$ & & $12(52)$ & & $7(58)$ \\
\hline $\mathrm{R} 1$ & & $9(38)$ & & $11(48)$ & & $5(42)$ \\
\hline PSA-free survival (months) & $4.57 \quad(1-12)$ & & $25.0 \quad(14-50)$ & & $(37-65)$ & \\
\hline
\end{tabular}

Molecular markers will help us to move into the promising field of personalized medicine (PM) (5). The concept of PM is to utilize person's genetic information to best plot a course for an individual in terms of disease risk assessment, prevention, treatment or palliation. The era of PM in cancer management promises more efficient treatment based on individual 'tumor characterization' rather than 'tumor anatomical/morphological classification'. Subdividing patients into smaller groups will provide more accuracy of prognosis and treatment efficiency. Furthermore, treatment will be given to only a fraction of patients who will respond, while the others, who gain no clinical benefit, will not need to suffer from severe toxic sideeffects. A number of platforms have been developed for PM in cancer, as outlined in our recent review (6). miRNA profiling is an emerging tool for PM in cancer.

MicroRNAs (miRNAs) are short, single-stranded, noncoding RNAs (7) that are predicted to regulate up to $30 \%$ of all messengerRNAs (mRNAs) (8). miRNAs function mainly through post-transcriptional regulation of gene expression and participate in various physiological as well as pathological processes (9).

The suite of clinical applications of miRNA in cancer is broad, encompassing diagnostic, prognostic and predictive clinical applications. miRNAs can also serve as predictive indicators of disease relapse and metastasis. miRNAs have been shown to be dysregulated in the majority of solid tumors, including kidney $(10,11)$ and bladder $(12)$. Several reports have documented dysregulation of miRNAs between normal and cancerous tissues in prostate cancer (13-19). Moreover, miRNA expression is related to tumor stage and progression $(16,20)$, perineural invasion (21) and androgen-dependence $(15,20,22)$. Recent reports indicate that miR-221 and miR-96 can predict biochemical recurrence $(17,23)$.

In this study we aimed using a low density real-time quantitative polymerase chain reaction (RT-qPCR) assay to identify miRNAs that are differentially regulated in prostate cancer patients having an early biochemical relapse, late biochemical relapse or no biochemical relapse. In order to use miRNAs as potential prognostic markers for predicting a recurrence of prostate cancer after radical prostatectomy, we evaluated in this respect as first proof-of-principle two of the most striking miRNAs, miR-10b and miR-222, by quantitative RT-qPCR. Since in silico and experimental evidence indicates that miRNAs can influence cell proliferation and migration and that their predicted targets function in tumor progression, we assessed their potential biological significance by testing the effect of miR-10b on these cellular characteristics.

\section{Materials and methods}

Tissue specimen and cell culture. Tissues from 52 primary prostate cancers were obtained after RP between 2001 and 2008 at Charité, University Hospital, Berlin, Germany and St. Michael's Hospital, Toronto, Canada. Tissues were routinely formalinfixed and paraffin-embedded (FFPE) directly after surgery. To obtain a pure tumor population, tumor areas were identified by haematoxylin/eosin staining and punch-biopsied with a 1-mm tissue microarray punch needle. Tumor content was re-confirmed by a pathologist. Patients were initially divided into three study groups for profiling: i) patients with an early 
biochemical relapse ( $<1$ year after $\mathrm{RP}, \mathrm{N}=10)$, ii) patients with a biochemical relapse between 1-4 years after $\mathrm{RP}, \mathrm{N}=10$ ) and patients with no biochemical relapse during the follow-up of at least 3 years $(\mathrm{N}=10)$ (Table I). For validation, samples were divided into two groups: i) patients with an early biochemical relapse $(<1$ year after $\mathrm{RP}, \mathrm{N}=24)$ and ii) patients with no or late biochemical relapse (at least 2 years after $\mathrm{RP}, \mathrm{N}=22$ ). The latter cohort included 22 of the 28 patients from the 2 patient groups characterized in the middle and right column of Table I, while all patients with a biochemical relapse between 1 and 2 years were excluded from further analysis. Biochemical relapse was defined as the first post-operative PSA of $>0.1 \mathrm{ng} / \mathrm{ml}$, as confirmed by at least 1 subsequent increasing value (persistent PSA increase) after achieving undetectable PSA post-operatively, defined as a detection limit of $<0.04 \mathrm{ng} / \mathrm{ml}$. Patients with no relapse were censored at the time-point of the last request.

LNCaP and DU-145 prostate cancer cell lines were grown in RPMI-1640 medium supplemented with $10 \%$ fetal calf serum at $37^{\circ} \mathrm{C}$ in a humidified $5 \% \mathrm{CO}_{2}$ atmosphere.

Total RNA extraction. Total RNA extraction from malignant FFPE tissue was performed using the Recover All total nucleic acid extraction kit (Ambion, Austin, TX, USA), following the manufacturer's protocol. FFPE tissue was minced with a scalpel and deparaffinized. Prior to protease digestion the tissue was homogenized using a TissueLyser (Qiagen, Hilden, Germany). After protease digestion, samples were demodified by adding $26.5 \mu 15 \mathrm{M} \mathrm{NH}_{4} \mathrm{Cl}$ and incubating the sample at $94^{\circ} \mathrm{C}$ for 20 min (24). RNA extraction of benign FFPE tissue was performed with the miRNAeasy FFPE kit (Qiagen) according to the manufacturer's instructions.

The yield and quality of extracted RNA was assessed by spectrometrical analyses (ratio of 260-280 nm) with a NanoDrop 1000 Spectrophotometer (NanoDrop Technologies, Wilmington, DE, USA) and RNA integrity number was calculated on the Bioanalyzer 2100 (Agilent Technologies, Santa Clara, CA, USA).

Multiplex quantitative RT-PCR. Multiplex quantitative RT-PCR was performed using the TaqMan Array Human MicroRNA A+B Cards v3.0 (Applied Biosystems, Foster City, CA, USA) according to the manufacturer's instructions. A pool of 10 RNA samples was prepared for each relapse group using 400 ng RNA per sample. Pooled RNA (517 ng) were reverse transcribed using Megaplex RT primers A or B from the Megaplex Primer Pool, Human Pools Set v3.0 (Applied Biosystems) and the TaqMan MicroRNA Reverse Transcription kit (Applied Biosystems) according to the manufacturer's protocol. RT-qPCR was performed on the ABI 7900 machine (Applied Biosystems) in 40 cycles. Expression was normalized to RNU6B, which is provided as endogenous control on the arrays. $\mathrm{Cq}$ values above 32 were regarded as not expressed according to the manufacturer's recommendation.

Quantitative RT-PCR. Quantitative miRNA RT-PCR was performed with the TaqMan MicroRNA Assay kit according to the manufacturer's recommendations (Applied Biosystems). RT-PCR was performed for two dysregulated miRNAs (hsamiR-10b, hsa-miR-222) in a total of 46 cases as described previously (16). Briefly, $6.67 \mathrm{ng}$ total RNA was reversetranscribed into cDNA using gene-specific miRNA RT-qPCR primers followed by amplification for 40 cycles using ABI Step One System (Applied Biosystems) and miRNA specific probes (Applied Biosystems). Each experiment was done in triplicate. Expression values were normalized to RNU44. Standard curves and efficiencies were measured for each miRNA (miR-10b, $\mathrm{E}=1.99$; miR-130b, $\mathrm{E}=1.99$; miR-222, $\mathrm{E}=1.98$; RNU44, $\mathrm{E}=2.00$ ). Normalization was done as previously described (16).

Transfection of prostate cancer cell lines. LNCaP and DU145 were transfected with pre-miRNAs and anti-miRNA inhibitors (Applied Biosystems) for miR-10b in a final concentration of $10 \mathrm{nM}$ using siPort NeoFX transfection agent (Applied Biosystems). In all experiments a culture transfected with a negative control (NC) pre-miR (pre-NC\#1; Applied Biosystems) and a no transfection control were carried along.

Proliferation assay. Proliferation of pre-miR-10b transfected LNCaP cells was assessed by metabolic conversion of 3-(4,5-dimethylthiazol-2-yl)-2,5-diphenyl tetrazolium bromide (MTT) (Sigma Aldrich, Oakville, Ontario, Canada). Cells were grown to $80 \%$ confluence and seeded into 96 -well plates at a final concentration of $6 \times 10^{3}$ cells per well and transfected with $10 \mathrm{nM}$ precursor miRNA, anti-miRNA inhibitor or pre-miR-NC\#1. Cells were allowed to rest for $24 \mathrm{~h}$ and were then serum starved for another $24 \mathrm{~h}$. Cultures were incubated in $5 \mathrm{mg} / \mathrm{ml}$ MTT for $4 \mathrm{~h}$ and lysed in $10 \%$ SDS in $0.01 \mathrm{M} \mathrm{HCl}$. Lysates were incubated at $37^{\circ} \mathrm{C}$ overnight and absorbance of formazan was measured at $550 \mathrm{~nm}$. Each reaction was performed in triplicate and results are shown as average of three independent assays.

Wound-healing assay. To assess the migrative phenotype of DU-145 cells upon pre-miR-10b transfection we performed a wound-healing assay. Cells were grown to $80 \%$ confluence and seeded into 6-well plates and transfected with $10 \mathrm{nM}$ precursor-miRNA, pre-miR-NC\#1 or anti-miRNA inhibitor. After reaching confluence, the cells were scratched with a $100-\mu 1$ pipette tip. Re-migration of cells to the wound was assessed by life cell imaging for $24 \mathrm{~h}$ under serum starvation. Percentage of open area was measured with the software TScratch (25) and the velocity of migration or the open area relative to time-point 0 was assessed.

Bioinformatics analysis. Target prediction analysis was performed using miRecords (http://mirecords.biolead.org/). We applied strict inclusion criteria and considered a prediction only if it was concordantly identified by two of the three most common algorithms (miRanda, picTar, TargetScan) as well as two other algorithms.

Predicted targets were forwarded to gene ontology (GO) analyses using the Database for Annotation, Visualization and Integrated Discovery (DAVID, http://david.abcc.ncifcrf.gov/ home.jsp) to test for overrepresentation of functional identity. The over-representation of genes from the same pathway was analysed using Reactome Skypainter 2 (http://www.reactome. org/cgi-bin/skypainter2). Chromosomal localization of the miRNA and overlapping transcripts were identified using miRBase (http://www.mirbase.org/index.shtml). 
Table II. Differential expression of the top dysregulated miRNAs in prostate cancer relapse patients given as fold changes in comparison to the no-relapse cohort. ${ }^{\mathrm{a}}$

\begin{tabular}{lccc}
\hline miRNA & $\begin{array}{c}\text { No relapse } \\
\text { within 3 years }\end{array}$ & $\begin{array}{c}\text { Relapse } \\
>1-4 \text { years }\end{array}$ & $\begin{array}{c}\text { Relapse } \\
<1 \text { year }\end{array}$ \\
\hline hsa-miR-23a & NE $^{\text {b }}$ & $(89.44)$ & $(646.34)$ \\
hsa-miR-449a & NE & $(22.25)$ & $(247.59)$ \\
hsa-miR-449b & NE & $(68.46)$ & $(207.79)$ \\
hsa-miR-200a & 1.00 & 1.12 & 2.53 \\
hsa-miR-1233 & 1.00 & 2.23 & 2.36 \\
hsa-miR-10b & $\mathbf{1 . 0 0}$ & $\mathbf{2 . 5 3}$ & $\mathbf{1 . 9 0}$ \\
hsa-miR-1825 & 1.00 & 1.11 & 1.84 \\
hsa-miR-186 & 1.00 & 0.64 & 0.67 \\
hsa-miR-1275 & 1.00 & 1.08 & 0.65 \\
hsa-miR-532-5p & 1.00 & 0.99 & 0.64 \\
hsa-miR-193b & 1.00 & 1.10 & 0.64 \\
hsa-miR-886-3p & 1.00 & 1.13 & 0.60 \\
hsa-miR-664 & 1.00 & 1.29 & 0.58 \\
hsa-miR-196b & 1.00 & 0.45 & 0.58 \\
hsa-miR-1274B & 1.00 & 1.19 & 0.57 \\
hsa-miR-720 & 1.00 & 1.19 & 0.51 \\
hsa-miR-146b-5p & 1.00 & 0.83 & 0.49 \\
hsa-miR-222 & $\mathbf{1 . 0 0}$ & $\mathbf{0 . 8 5}$ & $\mathbf{0 . 4 4}$ \\
hsa-miR-31 & 1.00 & 0.91 & 0.43 \\
hsa-miR-127-5p & 1.00 & 0.27 & NE \\
\hline
\end{tabular}

${ }^{a} \mathrm{~A}$ full list of differentially expressed miRNAs are available upon request. ${ }^{\mathrm{b}} \mathrm{NE}$, not expressed.

Statistical analysis. Statistical tests were performed with GraphPad Prism 5.04 (GraphPad Software Inc., San Diego, CA, USA), PASW Statistics 18.0.0 (IBM Corp., Somers, NY, USA) or Medcalc 11.5.1.0 (MedCalc Software, Mariakerke,
Belgium). Kruskal-Wallis test and Spearman rank correlation coefficients were used. All tests were performed two-tailed and p-values $<0.05$ were considered statistically significant. Receiver operating characteristic (ROC) curves were calculated to determine the potential of miRNAs to discriminate between recurrent and non-recurrent samples. Kaplan-Meier analysis and multivariate Cox regression were performed to identify variables associated with biochemical recurrence.

\section{Results}

Patient and tumor characteristic. miRNA expression analysis was performed in 52 prostate cancer specimen. Patient and tumor characteristics are summarized in Table I.

Identification of differentially expressed miRNAs in prostate cancer relapse. To address the hypothesis that miRNAs can be regulated in prostate cancer relapse, we performed a Megaplex RT-qPCR for three pooled RNA samples, that correspondent to a group of early relapse, late relapse and no relapse patients as defined in the material section. In total, 198 of 762 miRNAs showed a detectable signal (>32 Cq) in at least one pool. A miRNA was defined to be differentially expressed if the fold change in comparison to the no relapse pool was $> \pm 1.5$. According to this definition, 63 miRNAs were differentially expressed (Table II), of these $35 \mathrm{miRNAs}$ were up-regulated and 28 miRNAs down-regulated. We performed a literature search for the 63 miRNAs and thus identified 13 miRNAs (miR-10b, miR-30d, miR-31, miR-133b, miR-135a, miR-143, miR-151-3p, miR-200a, miR-221, miR-222, miR-375, miR-429, miR-516-3p), which have been described as putative prognostic or predictive markers in cancer (Table III).

Validation of dysregulated miRNAs. The array results are a proof-of-principle that miRNA might be differentially expressed in prostate cancers that relapse in comparison to non-relapsing tumors. We validated expression of two miRNAs, miR-10b and miR-222 in 46 prostate cancer specimen (Fig. 1A and B). miRNAs for validation were chosen according to the following criteria: i) at least 1.5-fold expression change, ii) expression

Table III. miRNAs with a previously described prognostic significance in cancer.

\begin{tabular}{|c|c|c|}
\hline miRNA & Prognostic significance & Refs. \\
\hline $\operatorname{miR}-516-3 p$ & Correlated with pathological grade, associated with ER(+)/LNN breast tumor aggressiveness & $(36)$ \\
\hline miR-200a & Associated with survival in cervical tumors & $(37)$ \\
\hline $\operatorname{miR}-10 b$ & Predicts survival in gastric cancer & $(38)$ \\
\hline $\operatorname{miR}-429$ & Associated with prognosis in ovarian cancer & $(39)$ \\
\hline miR-30d & Associated with overall survival in NSCLC & $(40)$ \\
\hline $\operatorname{miR}-375$ & Associated with prognosis in esophageal cancer with Barrett's & $(41)$ \\
\hline $\operatorname{miR}-143$ & Correlated with tumor stage in prostate cancer and tumor size in colorectal cancer & $(42,43)$ \\
\hline miR-151-3p & Associated with CLL harboring 17 p deletion and CLL with IGHV unmutated genes & $(44)$ \\
\hline $\operatorname{miR}-222$ & Associated with poor outcome in pancreatic tumors & $(45)$ \\
\hline miR-31 & Correlated with Gleason score in prostate cancer and invasion in colorectal cancer & $(16,46)$ \\
\hline $\operatorname{miR}-135 \mathrm{a}$ & Associated with relapse and disease-free survival in $\mathrm{cHL}$ & $(47)$ \\
\hline $\operatorname{miR}-221$ & Associated with biochemical recurrence in prostate cancer & $(17)$ \\
\hline $\operatorname{miR}-133 b$ & Associated with prognosis in bladder cancer & $(48)$ \\
\hline
\end{tabular}



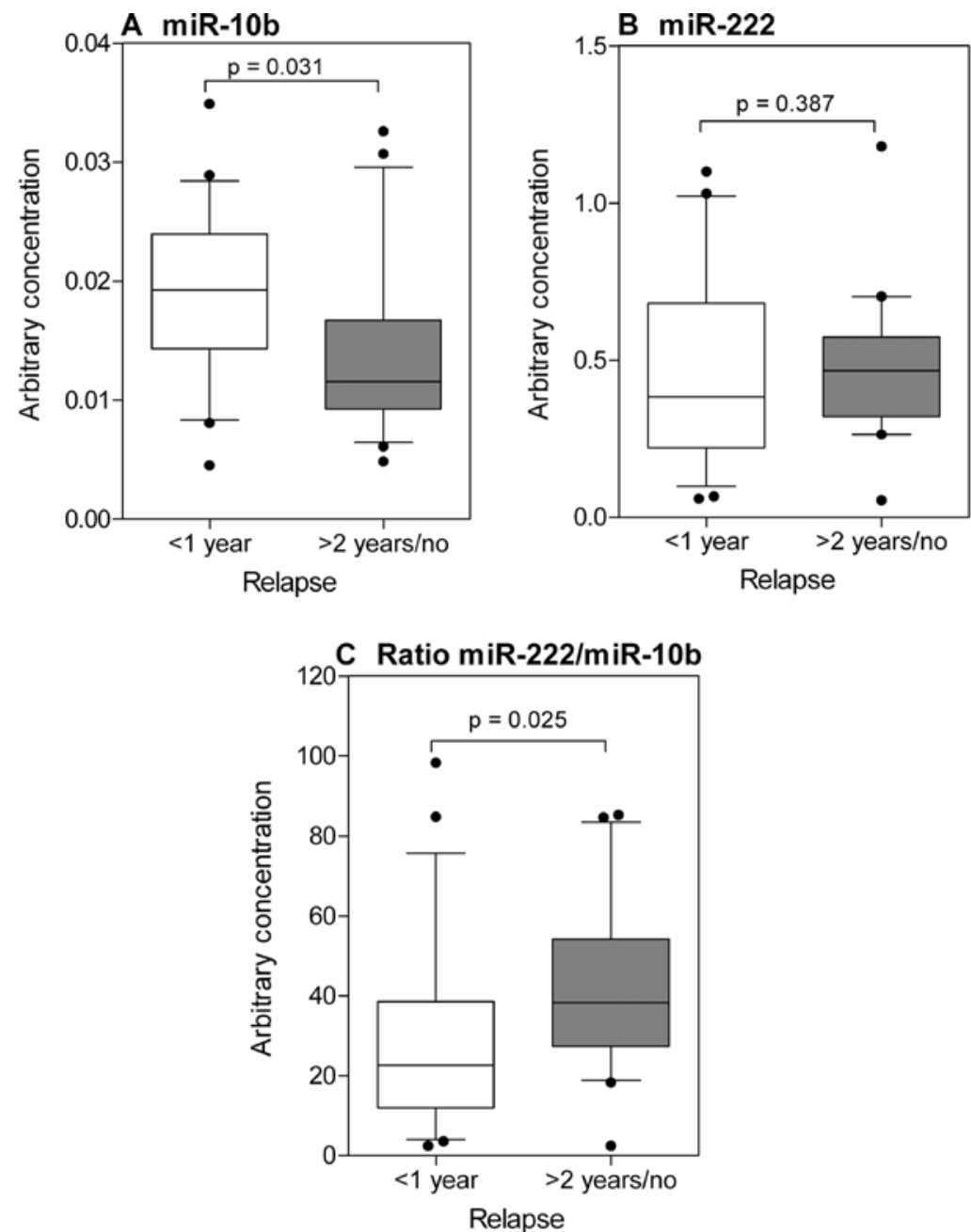

Figure 1. Expression of (A) miR-10b, (B) miR-222 expression and (C) expression ratio of miR-222/miR-10b. Boxes represent the lower and upper quartiles with medians, whiskers the 10 and 90 percentiles. miRNA expression was measured by TaqMan RT-qPCR. Expression was normalized to the geometric mean of miR-130b and RNU44, interplate controls and RT-qPCR efficiency. Significances, calculated by the Kruskal-Wallis test, ${ }^{*} \mathrm{p}<0.05$.

values below $30 \mathrm{Cq}$ values and iii) description of regulation in progression/metastases in another malignancy.

miR-10b showed a 1.67-fold higher median expression in the patients with a biochemical relapse in $<1$ year in comparison to patients with no biochemical relapse for at least 2 years (Fig. 1A; Mann-Whitney test, $\mathrm{p}=0.031$ ). miR-222 showed a slightly lower median expression of 1.21-fold, this trend was not statistically significant (Fig. 1B; Mann-Whitney, $\mathrm{p}=0.387$ ). The expression ratio of miR-222 to miR-10b was calculated to enhance the power of the variates to predict biochemical relapse. The miR-222/miR-10b ratio was 1.69 -fold lower in the patients with a relapse in $<1$ year (Fig. 1C; Mann-Whitney test, $\mathrm{p}=0.025$ ).

Association of miRNAs to clinicopathological parameters and biochemical relapse. miR-10b did not correlate with the histological grade (Gleason score), the tumor stage and the surgical margin, while miR-222 was inversely correlated with the histological grade (Table IV). Also, the miR-222/miR-10b ratio was significantly correlated with histological grade $\left(r_{S}=0.41\right.$, $\mathrm{p} \leq 0.01)$ and tumor stage $\left(\mathrm{r}_{\mathrm{S}}=-0.31, \mathrm{p}=0.04\right)$.

To investigate the capacity of the dysregulated miRNAs to discriminate patients with a biochemical relapse in $<1$ year from the remaining patients, we performed ROC analysis.
Table IV. Correlation of miRNA expression with biochemical relapse and clinicopathological parameters.

\begin{tabular}{lcccccc}
\hline & \multicolumn{2}{c}{ miR-10b } & & \multicolumn{2}{c}{ miR-222 } \\
\cline { 2 - 3 } \cline { 6 - 7 } \cline { 5 - 6 } & $\mathrm{r}_{\mathrm{S}}$ & $\mathrm{p}$-value & & $\mathrm{r}_{\mathrm{S}}$ & $\mathrm{p}$-value \\
\hline Gleason score & 0.24 & 0.11 & & -0.31 & 0.04 \\
Pathological stage & 0.17 & 0.28 & & -0.22 & 0.16 \\
Surgical margins & -0.02 & 0.92 & & 0.17 & 0.27 \\
Pre-operative PSA & 0.14 & 0.37 & & -0.08 & 0.91 \\
\hline
\end{tabular}

miR-10b and the miR-222/miR-10b showed a good discrimination with areas under the curve of 0.72 and 0.70 , respectivly, while miR-222 was not able to discriminate between the groups (AUC=0.56).

We then performed Kaplan-Meier analysis and multivariate Cox regression to determine, which miRNAs were significantly associated with biochemical relapse. miR-10b, miR-222, miR-222/miR-10b ratio and the pre-operative PSA were dichotomized according to their medians. The Gleason 
A miR-10b

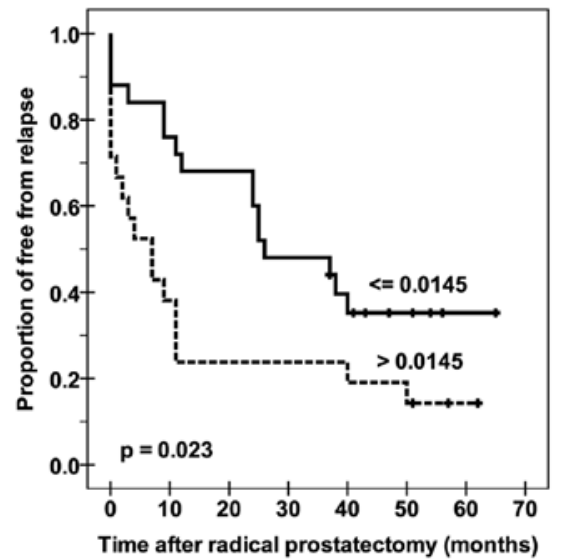

C Ratio of miR-222 to miR-10b

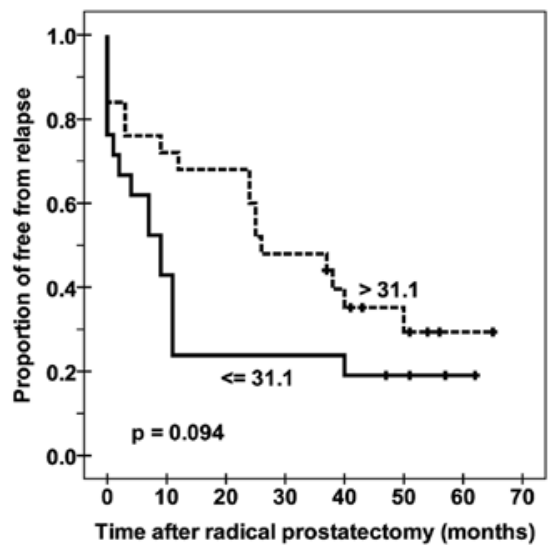

E Gleason score



B miR-222



D pT stage

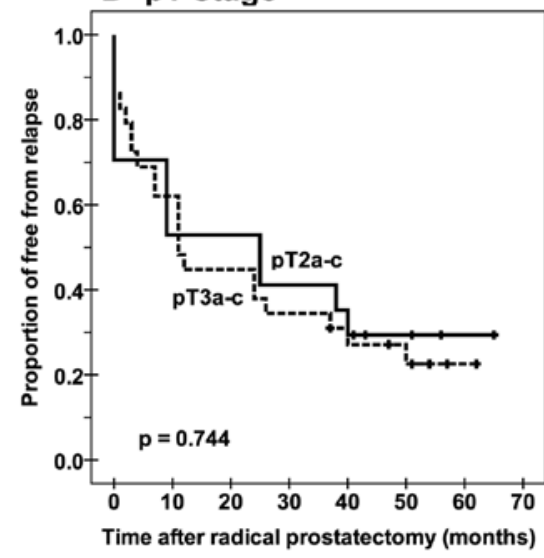

F Pre-operative PSA

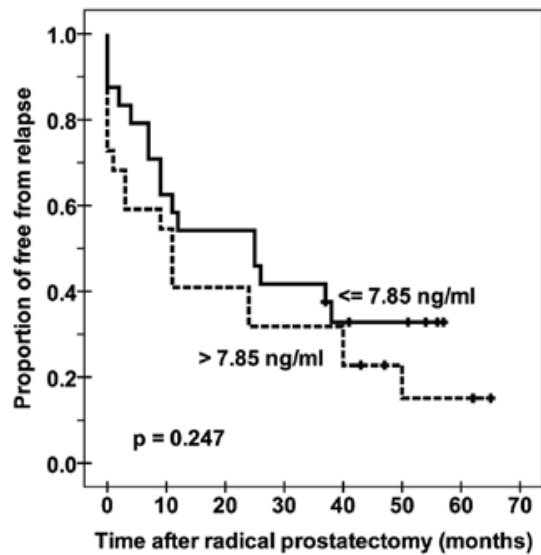

Figure 2. Kaplan-Meier analysis of recurrence-free survival according to various variables. Kaplan-Meier plots of (A) miR-10b, (B) miR-222, (C) ratio of miR-222 to miR-10b, (D) tumor stage, (E) Gleason score and (F) pre-operative PSA. The time of recurrence was defined as the first post-operative PSA value $>0.1 \mu \mathrm{g} / 1$ PSA confirmed by at least one subsequent rising value after the patients had reached an undetectable PSA level (detection limit $<0.04 \mu \mathrm{g} / \mathrm{l})$ after surgery.

score was trichotomized into a group of 6,7 , and above 7 . Pathological stage was dichotomized so that all samples with pT2 were grouped as well as all samples with pT3.

Kaplan-Meier analysis revealed a significant association of miR-10b expression with biochemical relapse (Fig. 2A; log-rank test, $\mathrm{p}=0.023)$, while miR-222 was not significantly associated with biochemical relapse (Fig. 2B). Surprisingly, none of the clinicopathological variables were significantly associated with biochemical relapse (Fig. 2D-F). In a multivariate Cox regression model, only miR-10b was an independent variable and remained the only variable in the model using forward and backward elimination methods (Table V).

Chromosomal location of regulated miRNAs. The 63 miRNAs are located on 67 chromosomal locations. Let-7f, miR-135a, miR-516b ${ }^{*}$ and miR-1233 were found in two different locations each. Twenty-eight miRNAs were intronic, while 35 miRNAs were intergenic. Two miRNAs were located in introns as well 
Table V. Cox regression of dysregulated miRNAs and clinicopathological variables.

\begin{tabular}{llcccc}
\hline Method & \multicolumn{1}{c}{ Variable } & $\beta$ & HR & 95\% CI & p-value \\
\hline Inclusion & miR-10b & 0.76 & 2.15 & $1.02-4.51$ & 0.044 \\
& Gleason score & -0.22 & 0.80 & $0.37-1-72$ & 0.575 \\
& Pathological stage & -0.11 & 0.90 & $0.40-1.99$ & 0.787 \\
& Surgical margins & 0.07 & 1.07 & $0.50-2.28$ & 0.864 \\
Forward LR & Pre-operative PSA & 0.25 & 1.28 & $0.64-2.58$ & 0.485 \\
Backward LR & miR-10b & 0.74 & 2.10 & $1.06-4.17$ & 0.033 \\
& miR-10b & 0.74 & 2.10 & $1.06-4.17$ & 0.033
\end{tabular}

HR, hazard ratio; CI, confidence interval; LR, likelihood ratio elimination approach of the Cox regression.
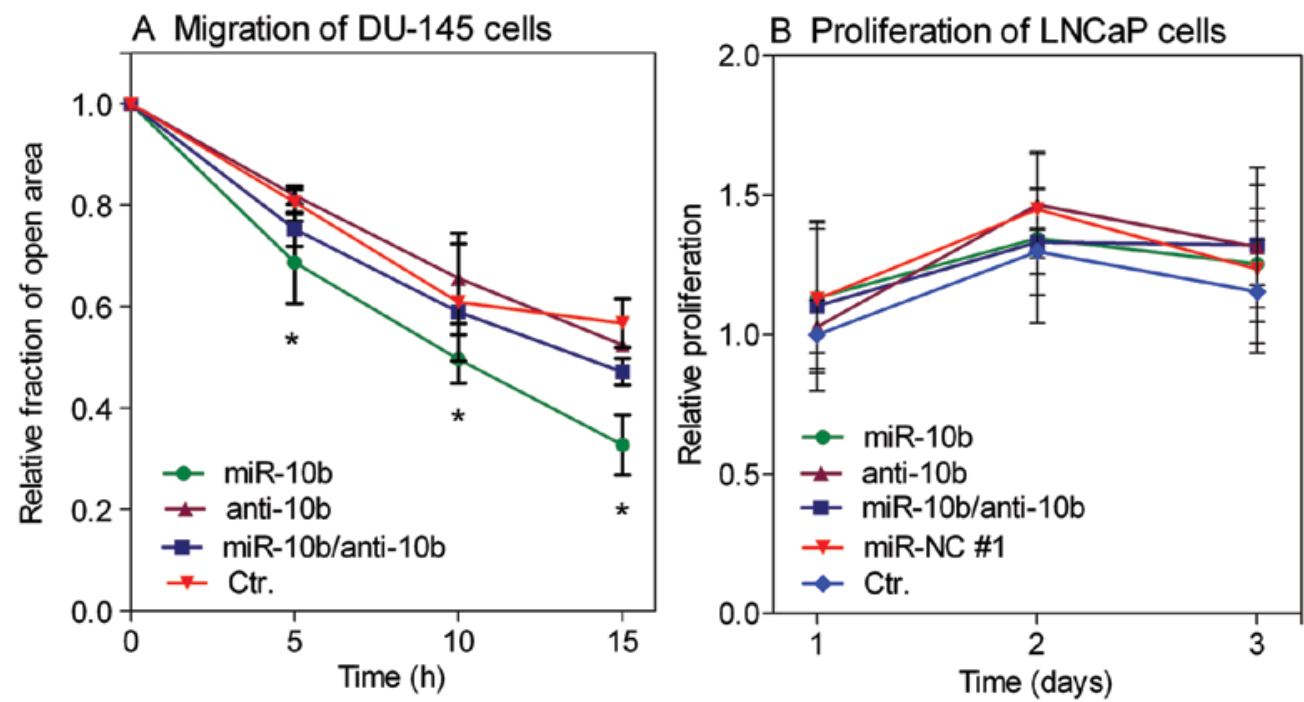

Figure 3. Effect of miR-10b on cell proliferation and migration. Cells were transfected with $10 \mathrm{nM}$ pre-miR-10b, anti-miR-10b, pre-miR-NC\#1 or the combination of pre-miR-10b and anti-miR-10b. (A) DU-145 cells were transfected and grown to 100\% density. Monolayers of the transfected and untreated (Ctr.) cells were scratched with a pipette tip and re-migration to the wound were analyzed for $15 \mathrm{~h}$ by life cell imaging. Pictures were taken every $10 \mathrm{~min}$. Percentage of open area was determined every $5 \mathrm{~h}$ using TScratch software. Data are presented as mean values from three independent assays ( \pm SD). Significance: Two-way ANOVA, ${ }^{*} p<0.05$. (B) The effect on cell proliferation of LNCaP cells was measured by MTT assay over three days. Mean values ( \pm SD) of three independent experiments. No statistical significance: Two-way ANOVA, $\mathrm{p}=0.732$.

as exons of splice-variants, while two miRNAs were only located in an exon.

We screened the set of differentially regulated miRNAs for expression clusters. A cluster was defined as a set of miRNAs that were located $<10 \mathrm{~kb}$ from each other. We identified 6 clusters whose members are dysregulated in prostate cancer relapse. Of them, 4 miRNA clusters show concordant expression.

In case of miR-126*, miR-151-3p, miR-196b and miR-449a and miR-449b a coexpression with their host genes (EGFL7, PTK2, HOXA9, CDC20b) has been described previously (26-30). Interestingly, the EGFL7 transcript, which hosts the miR-126 precursor, can be transcribed in three different variants each of them under control of its own promotor. Promotor studies identified one promotor, which is methylated in prostate cancer and treatment with methyltransferase and histone deacetylase inhibitors were able to up-regulate EGFL7 as well as miR-126 (29).
We further investigated if miRNAs are located in chromosomal aberration sites in prostate cancer relapse. A frequent chromosomal aberration in prostate cancer is $8 \mathrm{p}$ loss and $8 \mathrm{q}$ gain (31). The $8 \mathrm{q} 24.3$ region, which is amplified in prostate cancer, harbors miR-151-3p and miR-661.

Target prediction analysis and functional clustering of miRNA targets. To elucidate a potential function of the dysregulated miRNAs in prostate cancer relapse, we performed in silico target research using the software miRecords. Targets were filtered as described in Materials and methods. In total we identified targets for 36 miRNAs, ranging from 4-347 targets per miRNA (data available upon request). The predicted targets were used to perform gene ontology (GO) analysis using DAVID as well as pathway analysis using Reactome Skypainter.

Pathway analysis for miR-10b revealed, that mainly proteins involved in cell-cell junctions (ACTG1 and CADM1) and regulation of transcription (ESRRG, NR4A3, HNRNPK, 
GTF2H1, TBX5, NFAT5, HOXA1, KLF13) are over-represented in the set of samples. DAVID allows clustering of GO terms, which are concordantly identified in the sample. Again, regulation of transcription seems to be the most identified biological process in the set of target genes for miR-10b.

Reactome Skypainter analysis for miR-222 indicated that the regulation of protein stability (EIF5A2, SEMA6D, EIF3J) and the TRKA signaling pathway are over-represented in the set of target genes (CDKN1B and RALA). These results are in accordance with literature as CDKN1B is an experimentally proven miR-222 target (32). The most redundantly observed GO terms in DAVID analysis were related to regulation of transcription and apoptosis.

miRNAs dysregulated in relapse play a functional role in tumor progression. miR-10b showed a significant up-regulation of expression in patients with biochemical relapse. Therefore, we addressed the question how miR-10b influences tumor progression in vitro.

For wound-healing assays, we transfected DU-145 with $10 \mathrm{nM}$ pre-miR-10b, anti-miR-10b or combination of both and monitored remigration to the wound for $15 \mathrm{~h}$ (Fig. 3A). DU-145 cells transfected with pre-miR-10b showed a 70\% higher remigration to the wound in comparison to the transfection control (Fig. 3A; Two-way ANOVA, p<0.0001). The combined transfection of miR-10b and anti-miR-10 leads to a partial recovery from miR-10b function with only a $44 \%$ faster migration in comparison to the control, while transfection with anti-miR-10b has no significant effect on the remigration.

Proliferation was monitored by MTT assay (Fig. 3B). LNCaP cells were transfected with pre-miR-10b, anti-miR-10b, combination of both or pre-miR-NC\#1. After serum starvation, cell proliferation was monitored for 3 days. However, no significant effect on proliferation was observed (Fig. 3B; Two-way ANOVA, $\mathrm{p}=0.732$ ).

\section{Discussion}

This study is a proof-of-principle investigation for the utilization of miRNAs as predictors of biochemical relapse. Using pooled RNA from patients with i) early biochemical relapse, ii) late biochemical relapse and iii) no biochemical relapse we were able to show that a set of miRNAs is differentially expressed between the study groups. These results were validated for two miRNAs in a set of 46 prostate cancer specimens for miR-10b and miR-222.

FFPE tissue is routinely archived from all prostatectomized patients and is therefore the ideal sample for detection of prognostic markers. Expression profiling of mRNA fails to be reliable in FFPE tissue due to their high level of degradation in these tissue samples (33). miRNAs are in contrast to mRNAs considerably more stable in degraded RNA samples (34) and are reliably detectable in FFPE tissue, probably due to their small size and their cooperation into large ribonucleoprotein complexes (35). They might therefore be ideal markers for detection in FFPE tissue.

In prostate cancer relapse patients, 63 miRNAs were identified to be dysregulated between the groups. For a subset of 13 miRNAs (miR-10b, miR-30d, miR-31, miR-133b, miR-135a, miR-143, miR-151-3p, miR-200a, miR-221, miR-222, mIR-375,
miR-429, miR-516-3p) a previous deregulation in tumor progression or metastasis has been described in cancer $(16,17,36-48)$. Of them 6 miRNAs (miR-31, miR-133b, miR-143, miR-221, miR-222 and miR-375) have been previously described to be dysregulated in prostate cancer $(13,15,17,18,49,50)$. Therefore the deregulation of these miRNAs might be highly interesting in terms of biochemical relapse and should be further investigated in future studies.

As the results are based on the measurement of three pools from ten samples each, the risk of type I and II errors is high. The profiling step therefore clearly needs to be regarded as a proofof-principle. To confirm that miRNAs are indeed regulated in prostate cancer, we confirmed expression of two miRNAs in 46 prostate cancer samples in patients, who experienced a biochemical relapse in $<1$ year $(\mathrm{N}=24)$ and patients, who experienced no or late biochemical relapse ( $>2$ years after surgery; $\mathrm{N}=22$ ). Expression analysis by RT-qPCR confirmed the up-regulation of miR-10b and revealed a trend to a lower expression of miR-222 in patients with early biochemical relapse. These results support the hypothesis that miRNAs can predict biochemical relapse. Statistical analysis was performed to assess whether those miRNAs can separate patients with early biochemical relapse from the other patients. As is to be expected, miR-222 does not differentiate patients well. Yet, $\mathrm{miR}-10 \mathrm{~b}$ as well as miR-222/miR-10b expression ratio were comparably good discriminators with AUCs of approximately 0.70 . Kaplan-Meier confirmed the association of miR-10b with biochemical relapse. Interestingly, none of the clinicopathological variables was able to distinguish early relapse patients. Expression of miR-10b is not correlated with the clinicopathological parameters and it is an independent marker of biochemical relapse in a multivariate Cox regression model. Therefore, miR-10b expression might be an ideal prognostic marker that can accurately detect patients with biochemical relapse, especially where the available clinicopathological data fail to correctly classify patients.

This is the first study describing a deregulation of miR-10b in prostate cancer relapse. miR-10b has been described in relation to tumor progression and metastases in several tumor entities. It has been reported to be overexpressed in metastatic breast cancer (51), is further associated with high grade gliomas (52), and is higher expressed in hepatocellular carcinoma in comparison to adenocarcinomas (53). It is also part of a seven miRNA signature predicting survival in gastric cancer (38). A comparison of primary and metastatic tumors of breast, bladder, lung and colon revealed miR-10b to be overexpressed in bladder transitional cell carcinomas (54). Expression ratio of miR-221/222 was reported to be negatively associated with poor survival in ovarian cancer (55) and high miR-222 expression is associated with poor survival in pancreatic cancer (45) and invasive tumors in urothelial carcinomas (56). Thus, previous studies in other cancer entities support the regulation of miR-10b and miR-222 in tumor progression.

In silico target analysis was performed for all dysregulated miRNAs in prostate cancer relapse patients and we identified targets for 36 miRNAs. Analysis was further narrowed down on characterization of miR-10b, which is up-regulated in patients with biochemical relapse. Our in silico results indicate that miR-10b mainly targets genes involved in transcriptional regulation. We further transfected DU-145 prostate cancer 
cells with miR-10b precursor and identified a role of miR-10b in promoting migration of prostate cancer cells. Function of miR-10b has mainly been studied in breast cancer. In vitro it has been shown to promote migration and invasion in breast and esophageal cancer cell lines, as well as malignant peripheral nerve sheath tumor cell lines $(51,57,58)$. In vivo studies also highlight the importance of miR-10b in metastasis, since mice treated systemically with antagomiR-10b developed less metastasis than their non-treated counterparts (59). Several targets for miR-10b repression have been identified which further pinpoint the central role of miR-10b in migration and invasion. miR-10b directly targets Hoxd10 (60), a transcription factor, thereby regulating important pro-metastatic genes as RhoA, $\mathrm{RhoC}$ and urokinase-type plasminogen activator receptor (61). miR-10b further targets KLF4 another regulator of migration (57) and activates Ras-signaling (58).

In silico analysis of predicted miR-222 targets suggest a function of this miRNA in TRKA signaling. The direct binding of miR-221 and miR-222 to CDKN1B is experimentally proven in prostate cancer, thereby supporting the in silico results (32). miR-221 and miR-222 are strongly up-regulated in androgen-independent cells and induce androgen-independent growth and lower the response of the PSA-promotor to $5 \alpha$-DHT treatment (62). Expression of this miRNA cluster is not only dependent on androgen status, but can also be regulated by concordant binding of c-Jun and NF- $\kappa \mathrm{B}$ to its promotor (63). The miR-221/miR-222 cluster functions mainly in cell cycle transition (32) and promotes tumor growth in mice (64).

In conclusion, we present here the first proof-of-principle study that miRNAs are regulated in prostate cancer patients experiencing a biochemical relapse. Only few studies have before addressed the question of dysregulated miRNA in biochemical relapse, yet they studied only a small number of RNAs and provided only indecisive results $(18,65)$. We further show that the detection of differentially expressed miRNAs is a suitable approach and that these miRNAs might serve as prognostic markers for patients with an early biochemical relapse to provide them with closer follow-up and earlier treatment.

\section{Acknowledgements}

A.F. and K.J. were supported by the Foundation for Urologic Research, Berlin and the Sonnenfeld-Stiftung, Berlin. G.M.Y. was supported by Prostate Cancer Canada (grant no. 2010-555).

\section{References}

1. Amling CL, Blute ML, Bergstralh EJ, Seay TM, Slezak J and Zincke H: Long-term hazard of progression after radical prostatectomy for clinically localized prostate cancer: continued risk of biochemical failure after 5 years. J Urol 164: 101-105, 2000.

2. Han M, Partin AW, Zahurak M, Piantadosi S, Epstein JI and Walsh PC: Biochemical (prostate specific antigen) recurrence probability following radical prostatectomy for clinically localized prostate cancer. J Urol 169: 517-523, 2003.

3. Jhaveri FM, Zippe CD, Klein EA and Kupelian PA: Biochemical failure does not predict overall survival after radical prostatectomy for localized prostate cancer: 10 -year results. Urology 54: 884-890, 1999

4. Lughezzani G, Briganti A, Karakiewicz PI, et al: Predictive and prognostic models in radical prostatectomy candidates: a critical analysis of the literature. Eur Urol 58: 687-700, 2010.

5. Aggarwal BB, Danda D, Gupta S and Gehlot P: Models for prevention and treatment of cancer: problems vs promises Biochem Pharmacol 78: 1083-1094, 2009.
6. Arsanious A, Bjarnason GA and Yousef GM: From bench to bedside: current and future applications of molecular profiling in renal cell carcinoma. Mol Cancer 8: 20, 2009.

7. Berezikov E, Guryev V, van de Belt J, Wienholds E, Plasterk RH and Cuppen E: Phylogenetic shadowing and computational identification of human microRNA genes. Cell 120: 21-24, 2005.

8. Filipowicz W, Bhattacharyya SN and Sonenberg N: Mechanisms of post-transcriptional regulation by microRNAs: are the answers in sight? Nat Rev Genet 9: 102-114, 2008.

9. Metias SM, Lianidou E and Yousef GM: MicroRNAs in clinical oncology: at the crossroads between promises and problems. J Clin Pathol 62: 771-776, 2009.

10. Chow TF, Youssef YM, Lianidou E, et al: Differential expression profiling of microRNAs and their potential involvement in renal cell carcinoma pathogenesis. Clin Biochem 43: 150-158, 2010.

11. Jung M, Mollenkopf HJ, Grimm C, et al: MicroRNA profiling of clear cell renal cell cancer identifies a robust signature to define renal malignancy. J Cell Mol Med 13: 3918-3928, 2009.

12. Gottardo F, Liu CG, Ferracin M, et al: Micro-RNA profiling in kidney and bladder cancers. Urol Oncol 25: 387-392, 2007.

13. Ambs S, Prueitt RL, Yi M, et al: Genomic profiling of microRNA and messenger RNA reveals deregulated microRNA expression in prostate cancer. Cancer Res 68: 6162-6170, 2008.

14. Ozen M, Creighton CJ, Ozdemir M and Ittmann M: Widespread deregulation of microRNA expression in human prostate cancer. Oncogene 27: 1788-1793, 2008.

15. Porkka KP,Pfeiffer MJ, Waltering KK, Vessella RL, Tammela TL and Visakorpi T: MicroRNA expression profiling in prostate cancer. Cancer Res 67: 6130-6135, 2007.

16. Schaefer A, Jung M, Mollenkopf HJ, et al: Diagnostic and prognostic implications of microRNA profiling in prostate carcinoma. Int J Cancer 126: 1166-1176, 2010.

17. Spahn M, Kneitz S, Scholz CJ, et al: Expression of microRNA221 is progressively reduced in aggressive prostate cancer and metastasis and predicts clinical recurrence. Int J Cancer 127: 394-403, 2010

18. Tong AW, Fulgham P, Jay C, et al: MicroRNA profile analysis of human prostate cancers. Cancer Gene Ther 16: 206-216, 2009.

19. Volinia S, Calin GA, Liu CG, et al: A microRNA expression signature of human solid tumors defines cancer gene targets. Proc Natl Acad Sci USA 103: 2257-2261, 2006.

20. Lin SL, Chiang A, Chang D and Ying SY: Loss of mir-146a function in hormone-refractory prostate cancer. RNA 14: 417-424, 2008.

21. Prueitt RL, Yi M, Hudson RS, et al: Expression of microRNAs and protein-coding genes associated with perineural invasion in prostate cancer. Prostate 68: 1152-1164, 2008.

22. Leite KR, Sousa-Canavez JM, Reis ST, et al: Change in expression of miR-let7c, miR-100, and miR-218 from high grade localized prostate cancer to metastasis. Urol Oncol 29: 265-269, 2011.

23. Schaefer A, Stephan C, Busch J, Yousef GM and Jung K: Diagnostic, prognostic and therapeutic implications of microRNAs in urologic tumors. Nat Rev Urol 7: 286-297, 2010.

24. Oberli A, Popovici V, Delorenzi M, et al: Expression profiling with RNA from formalin-fixed, paraffin-embedded material. BMC Med Genomics 1: 9, 2008

25. Geback T, Schulz MM, Koumoutsakos P and Detmar M: TScratch: a novel and simple software tool for automated analysis of monolayer wound healing assays. Biotechniques 46: 265-274, 2009.

26. Ding J, Huang S, Wu S, et al: Gain of miR-151 on chromosome 8q24.3 facilitates tumour cell migration and spreading through down-regulating RhoGDIA. Nat Cell Biol 12: 390-399, 2010.

27. Lize M, Pilarski S and Dobbelstein M: E2F1-inducible microRNA $449 \mathrm{a} / \mathrm{b}$ suppresses cell proliferation and promotes apoptosis. Cell Death Differ 17: 452-458, 2010.

28. Musiyenko A, Bitko V and Barik S: Ectopic expression of miR-126*, an intronic product of the vascular endothelial EGF-like 7 gene, regulates prostein translation and invasiveness of prostate cancer LNCaP cells. J Mol Med 86: 313-322, 2008.

29. Saito Y, Friedman JM, Chihara Y, Egger G, Chuang JC and Liang G: Epigenetic therapy up-regulates the tumor suppressor microRNA-126 and its host gene EGFL7 in human cancer cells. Biochem Biophys Res Commun 379: 726-731, 2009.

30. Schotte D, Lange-Turenhout EA, Stumpel DJ, et al: Expression of miR-196b is not exclusively MLL-driven but especially linked to activation of HOXA genes in pediatric acute lymphoblastic leukemia. Haematologica 95: 1675-1682, 2010. 
31. Nupponen NN, Kakkola L, Koivisto P and Visakorpi T: Genetic alterations in hormone-refractory recurrent prostate carcinomas. Am J Pathol 153: 141-148, 1998.

32. Galardi S, Mercatelli N, Giorda E, et al: miR-221 and miR-222 expression affects the proliferation potential of human prostate carcinoma cell lines by targeting p27Kip1. J Biol Chem 282: 23716-23724, 2007.

33. Macabeo-Ong M, Ginzinger DG, Dekker N, et al: Effect of duration of fixation on quantitative reverse transcription polymerase chain reaction analyses. Mod Pathol 15: 979-987, 2002.

34. Jung M, Schaefer A, Steiner I, et al: Robust microRNA stability in degraded RNA preparations from human tissue and cell samples. Clin Chem 56: 998-1006, 2010.

35. Hasemeier B, Christgen M, Kreipe H and Lehmann U: Reliable microRNA profiling in routinely processed formalin-fixed paraffin-embedded breast cancer specimens using fluorescence labelled bead technology. BMC Biotechnol 8: 90, 2008.

36. Foekens JA, Sieuwerts AM, Smid M, et al: Four miRNAs associated with aggressiveness of lymph node-negative, estrogen receptor-positive human breast cancer. Proc Natl Acad Sci USA 105: 13021-13026, 2008.

37. Hu X, Schwarz JK, Lewis JS Jr, et al: A microRNA expression signature for cervical cancer prognosis. Cancer Res 70: 1441-1448, 2010.

38. Li X, Zhang Y, Ding J, Wu K and Fan D: Survival prediction of gastric cancer by a seven-microRNA signature. Gut 59: 579-585, 2010.

39. Nam EJ, Yoon H, Kim SW, et al: MicroRNA expression profiles in serous ovarian carcinoma. Clin Cancer Res 14: 2690-2695, 2008.

40. Hu Z, Chen X, Zhao Y, et al: Serum microRNA signatures identified in a genome-wide serum microRNA expression profiling predict survival of non-small cell lung cancer. J Clin Oncol 28: 1721-1726, 2010.

41. Mathe EA, Nguyen GH, Bowman ED, et al: MicroRNA expression in squamous cell carcinoma and adenocarcinoma of the esophagus: associations with survival. Clin Cancer Res 15: 6192-6200, 2009.

42. Clape C, Fritz V, Henriquet C, et al: miR-143 interferes with ERK5 signaling, and abrogates prostate cancer progression in mice. PLoS One 4: e7542, 2009.

43. Slaby O, Svoboda M, Fabian P, et al: Altered expression of miR-21, miR-31, miR-143 and miR-145 is related to clinicopathologic features of colorectal cancer. Oncology 72: 397-402, 2007.

44. Visone R, Rassenti LZ, Veronese A, et al: Karyotype-specific microRNA signature in chronic lymphocytic leukemia. Blood 114: 3872-3879, 2009.

45. Greither T, Grochola LF, Udelnow A, Lautenschlager C, Wurl P and Taubert H: Elevated expression of microRNAs 155, 203, 210 and 222 in pancreatic tumors is associated with poorer survival. Int J Cancer 126: 73-80, 2010.

46. Wang CJ, Zhou ZG, Wang L, et al: Clinicopathological significance of microRNA-31, -143 and -145 expression in colorectal cancer. Dis Markers 26: 27-34, 2009.

47. Navarro A, Diaz T, Martinez A, et al: Regulation of JAK2 by miR-135a: prognostic impact in classic Hodgkin lymphoma. Blood 114: 2945-2951, 2009.

48. Dyrskjot L, Ostenfeld MS, Bramsen JB, et al: Genomic profiling of microRNAs in bladder cancer: miR-129 is associated with poor outcome and promotes cell death in vitro. Cancer Res 69: 4851-4860, 2009.

49. Szczyrba J, Loprich E, Wach S, et al: The microRNA profile of prostate carcinoma obtained by deep sequencing. Mol Cancer Res 8: 529-538, 2010.
50. Wach S, Nolte E, Szczyrba J, et al: MicroRNA profiles of prostate carcinoma detected by multi-platform miRNA screening. Int J Cancer: March 11, 2011 (Epub ahead of print).

51. Ma L, Teruya-Feldstein J and Weinberg RA: Tumour invasion and metastasis initiated by microRNA-10b in breast cancer. Nature 449: 682-688, 2007.

52. Sasayama T, Nishihara M, Kondoh T, Hosoda K and Kohmura E: MicroRNA-10b is overexpressed in malignant glioma and associated with tumor invasive factors, uPAR and RhoC. Int J Cancer 125: 1407-1413, 2009.

53. Ladeiro Y, Couchy G, Balabaud C, et al: MicroRNA profiling in hepatocellular tumors is associated with clinical features and oncogene/tumor suppressor gene mutations. Hepatology 47: 1955-1963, 2008

54. Baffa R, Fassan M, Volinia S, et al: MicroRNA expression profiling of human metastatic cancers identifies cancer gene targets. J Pathol 219: 214-221, 2009.

55. Wurz K, Garcia RL, Goff BA, et al: MiR-221 and MiR-222 alterations in sporadic ovarian carcinoma: relationship to CDKN1B, CDKNIC and overall survival. Genes Chromosomes Cancer 49: 577-584, 2010.

56. Veerla S, Lindgren D, Kvist A, et al: MiRNA expression in urothelial carcinomas: important roles of miR-10a, miR-222, miR-125b, miR-7 and miR-452 for tumor stage and metastasis, and frequent homozygous losses of miR-31. Int J Cancer 124: 2236-2242, 2009.

57. Tian Y, Luo A, Cai Y, et al: MicroRNA-10b promotes migration and invasion through KLF4 in human esophageal cancer cell lines. J Biol Chem 285: 7986-7994, 2010.

58. Chai G, Liu N, Ma J, et al: MicroRNA-10b regulates tumorigenesis in neurofibromatosis type 1. Cancer Sci 101: 1997-2004, 2010.

59. Ma L, Reinhardt F, Pan E, et al: Therapeutic silencing of miR-10b inhibits metastasis in a mouse mammary tumor model. Nat Biotechnol 28: 341-347, 2010.

60. Debernardi S, Skoulakis S, Molloy G, Chaplin T, Dixon-McIver A and Young BD: MicroRNA miR-181a correlates with morphological sub-class of acute myeloid leukaemia and the expression of its target genes in global genome-wide analysis. Leukemia 21: 912-916, 2007.

61. Bourguignon LY, Wong G, Earle C, Krueger K and Spevak CC: Hyaluronan-CD44 interaction promotes c-Src-mediated twist signaling, microRNA-10b expression, and RhoA/RhoC upregulation, leading to Rho-kinase-associated cytoskeleton activation and breast tumor cell invasion. J Biol Chem 285: 36721-36735, 2010.

62. Sun T, Wang Q, Balk S, Brown M, Lee GS and Kantoff P: The role of microRNA-221 and microRNA-222 in androgen-independent prostate cancer cell lines. Cancer Res 69: 3356-3363, 2009.

63. Galardi S, Mercatelli N, Farace MG and Ciafre SA: NF-kB and c-Jun induce the expression of the oncogenic miR-221 and miR-222 in prostate carcinoma and glioblastoma cells. Nucleic Acids Res: Jan 18, 2011 (Epub ahead of print).

64. Mercatelli N, Coppola V, Bonci D, et al: The inhibition of the highly expressed miR-221 and miR-222 impairs the growth of prostate carcinoma xenografts in mice. PLoS One 3: e4029, 2008.

65. Leite KR, Tomiyama A, Reis ST, et al: MicroRNA-100 expression is independently related to biochemical recurrence of prostate cancer. J Urol 185: 1118-1122, 2011. 